http://rev.med.panacea.unica.edu.pe

Rev méd panacea. 2013; 3(3): 82-86.

Recibido: 10 de Septiembre del 2013 | Aceptado: 03 de Noviembre del 2013 | Publicado: 31 de Diciembre del 2013

CONFICTOS DE INTERÉS: NO DECLARADOS

ARTÍCULO DE REVISIÓN

\title{
Actualización sobre las Enfermedades Pulmonares Intersticiales Difusas (E. P. I. D)
}

\author{
Rafael Torres-Godomar ${ }^{1 a}$
}

Facultad de Medicina. Universidad San Luis Gonzaga. Ica, Perú1

Profesor principala

\begin{abstract}
a presente revisión tiene por finalidad actualizar nuestros conocimientos acerca de una patología — que la estamos viendo cada vez con mayor frecuencia y que por sus características clínicas, fisiológicas, radiológicas é histopatológicas se presta a confusión. Se está dando a conocer la clasificación propuesta por un grupo de expertos internacionales así como nuevos conceptos sobre la patogenia y la manera de enfocar el diagnóstico y tratamiento de éstas entidades que a la fecha, superan las 200 enfermedades y que con un diagnóstico oportuno abrigamos la esperanza de mejorar el pronóstico y la calidad de vida de éstos pacientes.
\end{abstract}

Palabras clave: enfermedades pulmonares intersticiales difusas idiopáticas (fuente: DeCS BIREME).

Autor corresponsal: Rafael Torres Godomar. Correo electrónico: rafo_neumo@hotmail.com

\section{INTRODUCCIÓN}

Las EPID están constituidas por un grupo terísticas clínicas, radiológicas, fisiológicas y heterogéneo de enfermedades que afectan patológicas similares y que afectan difusapredominantemente el intersticio pulmonar mente el parénquima pulmonar, compro(espacio anatómico comprendido entre metiendo también las vías respiratorias termembranas basales del epitelio alveolar y minales y la vasculatura pulmonar $(1,2,3$, del endotelio capilar) que presentan carac- 15,17).

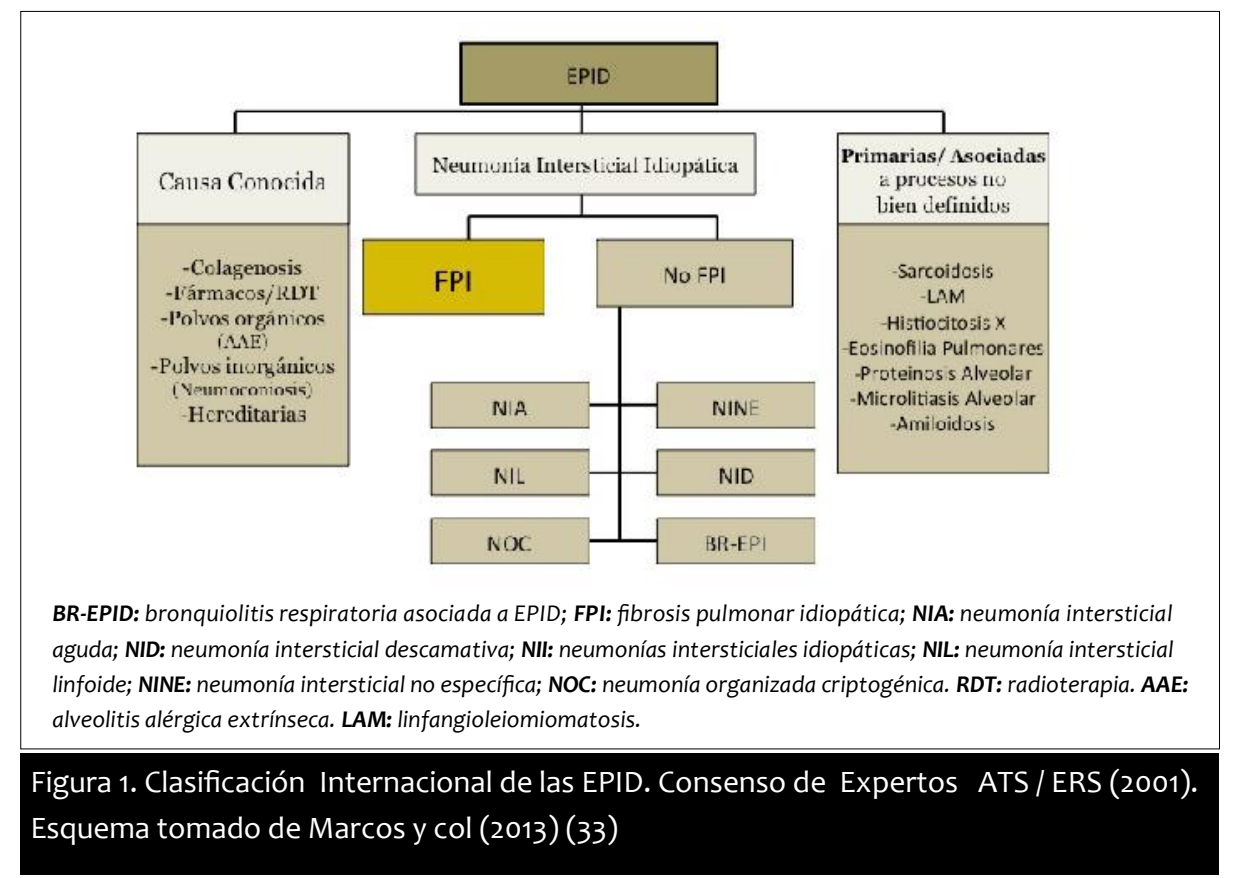

Tabla 1. Clasificación de las EPID, según el XXXII Congreso Neumosur 2006. Buenos Aires, Argentina

Neumonías Intersticiales Idiopáticas

Fibrosis Pulmonar Idiopática (FPI)

Neumonía Intersticial Aguda (NIA)

Neumonía Intersticial no específica (NINE)

Bronquiolitis respiratoria con EPID (BR/

EPID)

Neumonía Intersticial Descamativa

Neum. Organizada Criptogenética

Neumonía Intersticial Linfocítica

De causas conocidas o Asociadas

Asociadas a enfermedades del Colágeno

Causadas por polvos inorgánicos

(Neumoconiosis)

Inducidas por fármacos y radioterapia

Causadas por polvos orgánicos (alveolitis

alérgicas extrínsecas)

Asociadas a enf. Hereditarias (enf. De Hermanusky-Pudlak, etc).

Primarias o asociadas a otros procesos no bien definidos

Sarcoidosis

Proteinosis alveolar

Microlitiasis alveolar

Linfangioleiomatosis

Eosinofilias pulmonares

Histiocitosis X (granulomatosis de Cél.

Langerhans)

Amiloidosis

Otras EPID

\section{FISIOPATOLOGIA}

Los avances tecnológicos de los últimos años han permitido que la patogenia de las EPID esté en constante revisión. Clásicamente se ha considerado que al producirse 
la lesión del epitelio alveolar por el agente aggressor (conocido o no) se producía alveolitis, activándose un proceso inflamatorio con formación de exudado, edema y proliferación de células mesenquimales (linfocitos, macrófagos, PMN, eosinofilias y fibroblastos) que van a contribuir al engrosamiento del intersticio y generar mayor formación de colágeno alterando la membrana basal A-C.

Como la integridad del intersticio pulmonar es esencial para el intercambio gaseoso, su engrosamiento dificulta la difusión de los gases $\left(\mathrm{PaO}_{2}\right.$ y $\left.\mathrm{PaCO} 2\right)$. La hipoxemia resultante estimula el C.R. condicionando una hiperventilación que origina alcalosis respiratoria. En las fases más avanzadas de la enfermedad intervendrían fenómenos inmuno inflamatorios con liberación de proteasas y otras enzimas proteolíticas que favorecen la formación de tejido fibroso; la destrucción progresiva de las unidades alveolo capilares conducen a reducción del parénquima pulmonar, destrucción con dilatación de las vías aéreas y formación de cavidades quísticas, los capilares se ven obliterados por la fibrosis produciéndose hipertensión pulmonar.

Recientemente, Strieter y col.(8) han puntualizado que al producirse la ruptura de la membrana basal alveolo capilar, se distorsiona la arquitectura pulmonar y se acentúa la fibrosis con retracción del parénquima pulmonar. Todo ello contribuye a agravar la difusión gaseosa que progresivamente conduce a la insuficiencia respiratoria así como la hipertensión pulmonar conduce a cor pulmonar que son las causas principales de muerte en éstos pacientes. El final de las EPID es similar, cualquiera que sea su causa. (1, 3,24).

\section{APROXIMACIÓN DIAGNOSTICA A LAS EPID}

De Remee (9) y el Comité Expertos ATS/ ERS (4) nos dan conocer que para acercarnos al diagnóstico de estas enfermedades son necesarias la historia clínica, el examen físico, la radiografia de Tórax, la tomografía y el test de función pulmonar.

Historia Clínica: Es importante precisar los datos ocupacionales (tipo de ocupación y el tiempo de exposición). Generalmente los pacientes consultan por disnea progresiva al esfuerzo o por una tos persistente no productiva.

Si la disnea no es importante se debe pensar en Sarcoidosis, Histiocitosis $X$ o proteinosis alveolar, si el paciente acusa ortopnea se debe descartar insuficiencia ventricular

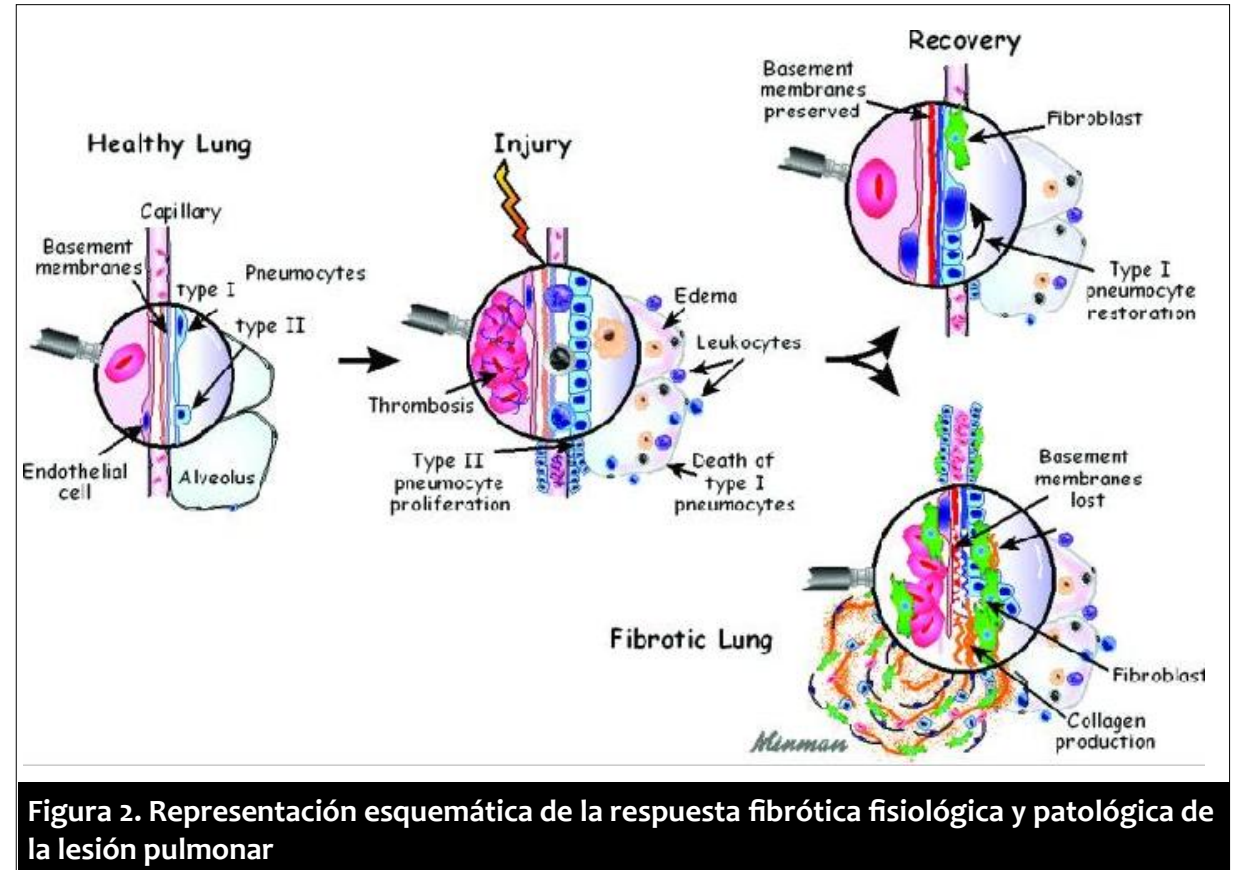

izquierda, si existiese exposición a drogas coidosis o neumoconiosis, de forma reticular como inmunosupresores (glucocorticoides o en la FPI y la asbestosis. Si es a predominio de citotóxicos). En caso de infecciones descartar las zonas medias o superiores considerar Pneumocystis jirovecii, micosis sistémicas y Sarcoidosis, Silicosis o Histiocitosis X. Si se micobacterias.

Tener en cuenta algún tipo de reacción pulmonar a algunos fármacos como las nitrofurantoínas, el uso de citostáticos puede causar toxicidad directa o inmunosupresión. Considerar la exposición ambiental al sílice, asbesto, berilio, polvos orgánicos o humos. Preguntar por antecedentes familiares de fibrosis quística, sarcoidosis o esclerosis tuberosa; asimismo, tener en consideracion antecedentes de neoplasias de mama, estómago, páncreas o pulmón.

Examen físico: Si a la auscultación hay presencia de estertores bibasales, sospechar de fibrosis pulmonar idiopática (FPI) o Asbestosis. Si se evidencia presencia de hipocratismo digital, sospechar de fibrosis pulmonar idiopática (FPI), Histiocitosis $\mathrm{X}$ o fibrosis quística. En caso de esplenomegalia, descartar Sarcoidosis.

Cuando se evidencia presencia de lesiones dérmicas, se debe sospechar de Sarcoidosis, Histiocitosis X, Lupus Eritematoso Sistemico (LES) o Dermatomiositis. En caso de adenopatías periféricas descartar Sarcoidosis o neoplasias. En presencia de Fiebre sospechar infecciones especialmente en pacientes inmunodeprimidos $(\mathrm{VIH})$.

Radiografia de Torax: Pueden mostrar imágenes de consolidación, de vidrio esmerilado en la fibrosis pulmonar idiopática (FPI), de aspecto quístico o nodulares en la sar- (Tabla 2). ven adenopatías hiliares puede tratarse de sarcoidosis o beriliosis. Si se observan calcificación hiliares "en cáscara de huevo" considerar sarcoidosis o silicosis.

Si se evidencia neumotórax descartar Histiocitosis $\mathrm{X}$, linfangioleiomatosis y esclerosis tuberosa. Si hubiera derrame pleural (quilotórax) considerar linfangioleiomatosis o enfermedades del colágeno. Cuando se ve calcificación pleural descartar asbestosis. Si en la imagen se observa un patrón de llenado alveolar se sugiere proteinosis alveolar, carcinoma a células, alveolares, insuficiencia cardiaca congestiva. Síndrome de distres respiratorio agudo, Síndrome de Goodpasture o Hemosiderosis pulmonar Idiopática. En cambio si la imagen es en panal de abejas cosiderar histiocitosis X, F P I (tardío).

En presencia de líneas de kerley sospechar de carcinoma linfangítico, insuficiencia cardiaca congestiva o linfangioleiomatosis. Si se evidencia un patrón miliar, sospechar de Tuberculosis, micosis (Histoplasmosis) o silicosis. La tomografía de alta resolución (TACAR) supera a la radiografía de tórax y es más precisa para el diagnóstico de estas entidades $(23,26)$.

El Dr. Reaño Ortega (10) en un trabajo presentado en la Sociedad Peruana de Neumologia (2011) dio a conocer un score que los radiólogos le asignaban a los hallazgos encontrados en la tomografía de alta resolución 
Tabla 2. Score utilizado por los radiólogos para el diagnóstico de FPI

\begin{tabular}{lll}
$\mathbf{P}$ & \multicolumn{1}{c}{ Alveolar } & \multicolumn{1}{c}{ Intersticial } \\
\hline $\mathbf{0}$ & Sin compromiso alveolar & Sin afección intersticial \\
$\mathbf{1}$ & Opacidad en vidrio esmerilado $<5 \%$ lóbulo & Engrosamiento septal sin panal de abejas \\
$\mathbf{2}$ & Opacidad en vidrio esmerilado $<25 \%$ lóbulo & Cambios en panal de abejas $<25 \%$ lóbulo \\
$\mathbf{3}$ & Opacidad en vidrio esmerilado $<25-49 \%$ lóbulo & Cambios en panal de abejas $<25-49 \%$ lóbulo \\
$\mathbf{4}$ & Opacidad en vidrio esmerilado $<50-75 \%$ lóbulo & Cambios en panal de abejas $<50-75 \%$ lóbulo \\
$\mathbf{5}$ & Opacidad en vidrio esmerilado $>75 \%$ del lóbulo & Cambios en panal de abejas $>75 \%$ del lóbulo
\end{tabular}

Se suma el puntaje para cada lóbulo y la suma de todos los lóbulos da puntaje final. Los autores concluyen que una puntuación mayor de dos en el patrón intersticial sumados a una edad avanzada (65 años) es altamente favorable para el diagnóstico de Fibrosis Pulmonar Idiopática. También concluyen que las variables fisiológicas incluyendo la capacidad vital forzada (FVC), la capacidad de difusión pulmonar para el monóxido de carbono (DLCO) y variable Prueba del esfuerzo de caminata por 6 minutos (6MWT) no son predictivas de diagnóstico de Fibrosis Pulmonar Idiopática $(10,11,12)$.

Pruebas de función pulmonar: lo característico es encontrar un patrón ventilatorio de tipo restrictivo con disminución de la capacidad de difusión, del monóxido de carbono, reducción uniforme de los volúmenes estáticos (capacidad vital y volumen residual), y en ausencia de obstrucción de vías aéreas. Cuando se asocian restricción más obstrucción se debe sospechar sarcoidosis, silicosis, histiocitosis $X$. Es frecuente que los pacientes con Sarcoidosis muestren función pulmonar normal con gasometría también normal.

Los niveles de gases en sangre en las EPID pueden ser normales en reposo pero se alteran con el ejercicio siendo característico de los trastornos de difusión encontrar hipoxia, hipocapnea y alcalosis respiratoria como ya se explicó anteriormente. El grado de anormalidad de la función pulmonar y de concentración de los gases arteriales estará en función de la severidad de la enfermedad intersticial.

\section{AYUDA DEL LABORATORIO}

Existen relativamente pocos test de utilidad para el diagnóstico de las EPID. En todos los casos se debe solicitar hemograma, urea, creatinina y electrolitos; examen del sedimento urinario y test de función hepática.

El médico puede solicitar algunos test en base a la sospecha clínica: anticuerpos antinucleares, dosaje de deshidrogenasa láctica $(\mathrm{DHL})$, en el $80 \%$ de éstos pactes se puede encontrar elevación en suero de enzima convertidora de angiotensina, la hipergamaglobulinemia policlonal, así como elevación del calcio en sangre y orina se encuentra en la Sarcoidosis; cuando se sospeche de hipersensibilidad específica por exposición se deben solicitar los exámenes serológicos apropiados.

La Gammagrafía con Galio-67 resulta positiva en FPI y la sarcoidosis (25). El Lavado broncoalveolar (LBA) es una técnica invasiva que utilizando Broncofibroscopio permite aspirar e identificar el número y las características de las células inmuno inflamatorias y son de utilidad para el diagnóstico así como para el pronóstico de la afección.

El Incremento de linfocitos estara presente en la sarcoidosis, beriliosis, neumonia por hipersensibilidad. El Incremento de polimorfonucleares se presentara en la neumonitis idiopáticas o sarcoidosis (etapa fibrótica), mientras que el Incremento de Eosinofilias, se presentara en la histiocitosis $\mathrm{X}$ y las eosinofilias pulmonares.

\section{CRITERIOS DIAGNOSTICOS DE LA FIBROSIS PULMONAR IDIOPÁTICA $(4,5,13,14)$.}

En pacientes con presencia de N I C en biopsia pulmonar, deben cumplirse los siguientes criterios:

- Exclusión de otras causas conocidas de EPID

- Alteración en función respiratoria: alteración ventilatoria restrictiva y/o alteración del intercambio de gases: aumento (A-a) $\mathrm{O}_{2}$ en reposo o con prueba de esfuerzo o en la disminución DLCO.

- Alteraciones típicas de la enfermedad en radiografia de tórax o TACAR.

En pacientes sin biopsia pulmonar deben cumplirse 4 criterios mayores y 3 criterios menores (Tabla 3).

\section{OBTENCIÓN DE MUESTRAS DE TEJIDO PARA EL DIAGNOSTICO DE LAS EPID \\ - Mediastinoscopía \\ - Biopsia pulmonar transbronquial \\ - Biopsia pulmonar abierta \\ - Misceláneo: Test de Kveim- Biopsia conjuntival o de piel \\ - Lavado bronco alveolar (estudio de célu- las y fluidos)}

Tabla 3. Criterios mayores y menores en

el diagnóstico de FPI en pacientes sin

biopsia pulmonar

Criterios Mayores

Exclusión de otras causas conocidas de

EPID

Alteraciones en la exploración funcional respiratoria ya señaladas

Alteraciones típicas de la enfermedad en Rx tórax o TACAR

Ausencia de alteraciones en biopsia transbronquial o en LBA que sugieran Dx alternativo

\section{Criterios Menores}

Edad superior a 50 años

Disnea de esfuerzo de comienzo insidio-

so, no explicada por otra causa

Duración de los síntomas superior a 3

meses

Estertores crepitantes bibasales, inspira-

torios y persistentes

\section{PRONOSTICO}

Es muy variable dependiendo de la enfermedad causal, así en la FPI el pronóstico es malo mientras que en la Sarcoidosis no lo es tanto. En la FPI se considera fatal ya que la sobrevivencia alcanza al 50\% después de los cinco años de iniciado el tratamiento. En las enfermedades autoinmunes 0 del colágeno el tiempo se sobrevida es relativamente corto

$(2,4,14,28,30)$

\section{TRATAMIENTO}

En la fase aguda de la enfermedad responden favorablemente a la administración de glucocorticoides (Prednisona o metil prednisolona $1 \mathrm{mg} / \mathrm{kg}$ VO por 6 a 8 semanas). En los casos de pobre respuesta se puede asociar otros inmuno supresores como Azotioprina o Ciclofosfamida también por VO Asimismo es recomendable el uso de $\mathrm{N}$-acetil cisteína como antioxidante. La oxigenoterapia ayuda a mejorar la calidad de vida de éstos pacientes. La rehabilitación pulmonar también es útil. La genoterapia es otra esperanza alentadora para éstos pacientes y en los casos muy avanzados la posibilidad de un trasplante pulmonar. $(2,4,13,14,15,20,32)$

\section{REFERENCIAS BIBLIOGRAFICAS}

1. Fishman Alfred P. Tratado de Neumología. Mc Graw-Hill, México Ed.1983 Vol 1:593- 759.

2. Wells AV, HIrani N. Intertisial Lung Disease Guideline Thorax 2008; 63(Suppl V):v1-v58. doi:10.1136/thx.2008.101691.

3. Harrison. Principios de Medicina Interna. Mc Graw-Hill Interamericana Ed 2012 Vol 2:21602169. 
3. American Thoracic Society, European Respiratory Society International. Multidisciplinary Consensus. Classification of the Idiopathic Interstitial Pneumonia. Am.J. Respir.Crit. Care Med. 2002; 165(2 ) : 277-304.

4. Rodríguez BE, López-Campos BJL, Vidal Serrano S. Diagnóstico y Tratamiento de las Enfermedades Pulmonares Intersticiales. Neumosur 2004; 16(2): 147-154.

5. Xanbet A. Ancochea J., Blanquer R. y col. Diagnóstico y Tratamiento de las Enfermedades Pulmonares intersticiales difusas. Arch. Bronconeumolog 2003; 39( 12 ): 580600.

6. Strieter RM Pathogenesis and natural history of usual interstitial pneumonia: the whole story of the last chapter of a long novel. Chest 2005; 128(suppl): $5268-5325$.

7. Strieter RM y Menhrod B. New mechanisms of Pulmonary Fibrosis. Chest 2009; 136: 1364 $-1370$.

8. De Remee RA. Diffuse Interstitial Pulmonary Disease from the Perspective of the Clinican.Chest 1987; 92(6): $1068-1073$.

9. Reaño RO (SPN) Clinical Predictors of Diagnosis of Idiopathic Pulmonary Fibrosis. Am.J.Resp.Crit. Care Med. Vol 181 (2010).

10. Feydya A, Sibila J, De Kerviler E. et al. Chest high resolution $C T$ in adults with primary humoral immunodeficiency. Br.J.Rad. 1996; 69: $1108-1116$.

11. Akira M, Hamada H, Sakatamy M. et al. CT finding during phase of accelerated deterioration in patients with idiopathic pulmonary fibrosis. AJR AM. J. Roentgenol 1997; 168: 79 $-83$.

12. Martinez FJ. Safrin S., Weyeker E. et al. The clinical course of patients with idiopathic pulmonary fibrosis. Ann.Intern.Med. 2005; 142: $963-967$.

13. Brown AW, Shlobin OA, Weir N. et al. A novel concept in idiopathic pulmonary fibrosis. Chest 2012; 142 (4): 1005- 1010.

14. Manual de Neumología (México 2008) Cap. 32 Enferm. Pulmonares Difusas. Fibrosis Pulmonar Idiopática : R. Vargas Gonzales, F. Canales Cid, JC Bioque Rivera, pag.363-371

15. 44th Annual Thomas L Petty Lung Conference: Pulmonary Genetics,Genomics and Gene Therapy. Chest 2002; 121:32-33 Suppl.

16. Escobedo Genebroso JW. Factores asociados a Enfermedades Intersticiales Difusas en adultos mayores hospitalizados en el servicio de medicina del hospital de EsSalud de Ica 2009-2010. Tesis para optar el titulo de especialista en medicina interna FMH "DAC" UNICA 2011

17. Perez-Padilla R, Salas J,Chapela R et al. Mortality in Mexican patients with chronic pigeon boleder's lung compared with those with usual interstitial pneumonia. Am. Rev. Resp. Dis. 1993; 148:49-53
18. Selman $M$, King TE, Pardo A. Idiopathic pulmonary fibrosis: prevailing and evolving hypothesis about its pathogenesis and implications for therapy. Ann Int Med 2001; 134:136-151

19. Ancochea J, Anton E,Casanova A. Nuevas estrategias terapeúticas en la fibrosis pulmonar idiopática. Arch Bronconeumol 2004; 40(6) : 16-22

20. Popa V, Colby TV, Reich SB. Pulmonary Interstitial Disease in Ig Deficiency. Chest 2002; 122: $1594-1603$

21. Miyazaki Y, Tateishi T et al. Clinical Predictors and Histologic Appearance of Acute exacerbations in chronic Hypersensitivity Pneumonitis Chest 2008; 134(6): 1265-1270

22. Hunninghake GW, Lynch DA, Galvin JR et al. Radiologic findings are strongly associated with a pathologic diagnosis of usual intersatitial pneumonia Chest 2003; 124: 1215- 1223

23. Liebow A, Carrington CB. Diffuse pulmonary lynphoreticular infiltrations associated with dysproteinemia. Med Clin North Am 1973; 37: 809- 821

24. Fernandez Perez ER, Daniels CE, Schroeder DR et al. Incidence, prevalence and clinical course of idiopathic pulmonary fibrosis: a population- based study. Chest 2010; 137(1): 129-137

25. Wescott JL, Cole SR. Traction bronchiectasis in end-stage pulmonary fibrosis. Radiology 1986; 161(3): 665- 669

26. Colland HR, King TE. Demystifynd idiopathic interstitial pneumonia. Arch Intern Med 2003; 163: 17-29

27. Park JH, Kim DS, Park IN et al. Prognosis of fibrotic interstitial pneumonia: idiopathic versus collagen vascular disease- related subtypes. Am J Respir Crit Care Med 2007 ; 175: 705-711

28. Strange C, Highland KB. Interstitial lung disease in the patient who has connective tissue disease. Clin Chest Med 2004; 25(3 ): 549-559

29. Fisher A, West SG, Swigris JJ et al. Connective Tissue Disease-Associated Interstitial Lung Disease. Chest 2010; 138(2 ): 251-256

30. Wells AV, du Bois RM. Bronchiolitis in association with connective tissue disorders. Clin Chest Med 1993; 4: 655- 665

31. Theodore AC, Tseng $\mathrm{CH}-\mathrm{H}$, Li $\mathrm{N}$ et al. Correlation of cough with disease activity and treatment with Cycophosphamide in Scleroderma interstitial lung disease. Chest 2012; 142 (3):614- 621

32. Marcos PJ, Montero C, Otero González I. Una mirada general a las enfermedades pulmonares intersticiales y una específica a la fibrosis pulmonar idiopática. Galicia Clin 2013;74(4):13-22. 
FINANCIAMIENTO: Autofinanciado.

AGRADECIMIENTOS: A los estudiantes de medicina de la facultad "Daniel Alcides Carrión" de la Universidad Nacional San Luis Gonzaga de Ica, Perú.

CONTRIBUCIONES DE AUTORÍA: RTG participo en el diseño del articulo, la revision crítica del mismo y la aprobación de la versión final.

\section{Update on Diffuse Interstitial Lung Disease (DILD)}

he revision aim is to update our knowledge about a pathology we find with higher frequency in our daily routine and confusion can be created according to the different clinical, physiological, radiologic, and histopathological features. We introduce a new classification method proposed by a group of experts foreign scientist, and new information about the pathogenesis, and the way to focus the diagnosis and treatment to this group of diseases that to date, exceed 2000 types. However. With a proper diagnostic, we hope to improve the prognosis and the quality of life for these patients.

Key words: interstitial lung disease (source: MeSH NLM).

CITA SUGERIDA.

Torres-Godomar R. Actualización sobre las Enfermedades Pulmonares Intersticiales Difusas (E. P. I. D). Rev méd panacea.2013; 3 (3):82-86. 